\title{
Endovascular Management of Chronic Mesenteric Ischemia
}

\author{
Mohamed Ismail, MD, Ramez Mounir Wahba, MD, Atef Abd Elhameed, MD \\ Department of Vascular Surgery, Ain Shams University, Cairo, Egypt
}

\begin{abstract}
Aim: The purpose of this study was to review the effectiveness and feasibility of endovascular intervention for chronic mesenteric ischemia and its effect on morbidity and mortality.

Patients and methods: Between march 2014, and October 2016, 7 patients diagnosed with chronic mesenteric ischemia had endovascular treatment. Demographic, clinical, diagnostic and procedure-related data were recorded, and strict follow up was done for 24 month postoperative.

Results: Data was obtained for 7 patients who underwent endovascular procedures during the study period. Superior mesenteric artery (SMA) was affected in all patients, inferior mesenteric in 3 patients $(42.9 \%)$ and celiac artery was affected in 2 patients $(28.6 \%)$ Technical success was achieved in 6 patients. SMA was the main target for revascularization. Technical success was achieved in 6 patients (85.7\%). 24 month Primary assisted patency was $71.4 \%$. There were no major complications, minor complications occurred in 2 patients $(28.6 \%)$.
\end{abstract}

Conclusion: Endovascular management of chronic mesenteric ischemia has comparable outcome to surgical solutions but with less morbidity and mortality. However strict follow up and timely secondary intervention is mandatory.

key words: Endovascular therapy, stent; chronic mesenteric ischemia, celiac artery, superior mesenteric artery, inferior mesenteric artery.

\section{Introduction}

Although chronic mesenteric ischemia (CMI) is rare, the incidence of mesenteric artery stenoses (MAS) is quite common, A number of relatively small studies showed an increasing prevalence with age going from $6 \%$ at age 40 , to $14 \%$ at 60 years, and between $18 \%$ and $67 \%$ in those over 75 years of age. These figures are comparable to those in peripheral artery disease $(\mathrm{PAD}){ }^{1}$

Chronic mesenteric ischemia develops from insufficient blood flow toward the gastrointestinal tract through the splanchnic vessels, mainly due to atherosclerotic stenosis. $^{2}$

Symptoms from this condition depend on the acuity of the pathologic process and may include abdominal pain, weight loss, or frank shock from intestinal infarction. ${ }^{3}$ However, patients with gastrointestinal or pancreatic malignancy may present with similar symptoms and some of these will also have significant atherosclerosis of the mesenteric vessels. It is therefore important to fully investigate these patients to exclude other pathology before attributing the symptoms to intestinal ischemia. $^{5}$

Duplex scanning of the mesenteric arteries is the ideal noninvasive test. However, when intervention is indicated, Computed tomography angiography (or Magnetic resonance angiography as an alternative) is the most useful investigation. Mesenteric angiography with lateral views to show the origins of the three visceral arteries is essential as a first step prior to endovascular treatment. ${ }^{5}$
Various lifestyle changes are recommended for patients with chronic mesenteric ischemia. However, despite smoking cessation and treatment of risk factors for atherosclerosis, many patients will require an invasive treatment, either percutaneous endovascular revascularization or open surgical revascularization. ${ }^{2}$

While surgical revascularization has been the standard treatment for symptomatic patients, recent advances in interventional devices and techniques have made endovascular treatment feasible and effective. Percutaneous transluminal angioplasty with stent placement is now recognized as a minimally invasive means of obtaining good long-term results. ${ }^{4}$

In patients with chronic mesenteric ischemia, nonrandomized data suggest that endovascular therapy reduces in-hospital complications and mortality while symptom relief and long-term patency are maintained. ${ }^{3}$

We sought to characterize our experience with endovascular treatment of mesenteric ischemia with emphasis on safety, procedural success, and long-term efficacy.

\section{Patients and methods}

Between March 2014, and October 2016, 7 patients presenting with chronic mesenteric ischemia received mesenteric revascularization at Ain Shams University hospitals.

For each patient, the following data were recorded: 
demographic data, such as patient anonymous identification number, sex, date of birth, and date of each procedure; patient medical history, such as history of diabetes, hypertension, current or past tobacco use, dyslipidemia, cardiac disease, peripheral arterial disease, history of stroke, chronic obstructive pulmonary disease, chronic kidney disease or renal insufficiency, gastric ulceration, hemoglobin level at the time of presentation, history of cancer, abdominal surgery, and current medications (at the time of the revascularization procedure); clinical symptoms leading to the diagnosis, such as postcibal angina, weight at the time of the first procedure, usual body weight (defined as the body weight 6 months before the procedure), and height, anemia (hemoglobin level less than $10 \mathrm{mg} / \mathrm{dl}$ ); diagnostic imaging and their findings, such as splanchnic vessels affected, stenosis or complete occlusion, length of the stenosis, cause of the stenosis, and type of stent used; and procedure-related data for each revascularization, such as immediate technical success, complications (local and systemic, procedure or anesthesia related), clinical outcome at the first follow-up visit (at 1 month), date and type of recurrence (clinical, angiographic, or both), total number of procedures, death during follow-up, and cause of death.

Inclusion criteria: All patients presenting with vague abdominal pain, postcibal angina, vomiting and/or weight loss due to a lesion (stenosis or occlusion) involving superior mesenteric and/ or coeliac arteries with no previous history of mesenteric open vascular or endovascular intervention.

Exclusion criteria: Patients presenting with Acute mesenteric ischemia, were excluded from our study.

All patients underwent preoperative evaluation including physical examination, full laboratory tests, pelviabdominal ultrasound, upper GIT endoscopy, mesenteric duplex and CT pelviabdominal with contrast and CT mesenteric angiography.

All procedures were performed in an operating theatre with vascular imaging. Access was obtained percutaneously through femoral or transbrachial access according to the angiographic feasibility. Hydrophilic guidewires (Terumo 0.035) and 0.018 (V18, Boston scientific) were used under fluoroscopic control with guidance of diagnostic catheters (Judkins right $6 \mathrm{~F}$ ), (Bern catheter 4 or $5 \mathrm{~F})$. The use of stents was tailored according to the patient's angiographic anatomy with the size and length determined according to the discretion of the operator with the preference of using selfexpanding stents (5-6 mm) except for ostial lesions where balloon mounted stents $(5-6 \mathrm{~mm})$ were preferably used.

Intraoperative angiography was performed to confirm satisfactory revascularization at the conclusion of each case.

Technical success was defined as residual stenosis $<30 \%$ on biplanar intraoperative arteriography. Clinical success was defined as the resolution of the postprandial abdominal pain previously experienced by the patient, and the absence of weight loss. Recurrence was defined as the reappearance of symptoms compatible with splanchnic syndrome, associated with significant vascular restenosis (using duplex ultrasound, computed tomography angiography, or arteriography) in at least one of the splanchnic vessels previously treated.

Complications were classified into major and minor. Major complications are those that led to mortality, increase in hospital stay, need for blood transfusion or secondary surgical intervention.

Our end point was improvement of the patient's condition which meant no need for further intervention to improve the patient's condition and assessment of primary patency and primary assisted patency Postoperative follow up was done immediately post procedure and at $1,3,6$, 12, 18 and 24 months thereafter. We assessed the improvement of the patient's complaint, gaining weight, and the need for further intervention to improve the patient's outcome.

The decision for further intervention was based upon no improvement or worsening of the patient's condition and this intervention was tailored according to the angiographic anatomy of the arterial tree of each patient. 


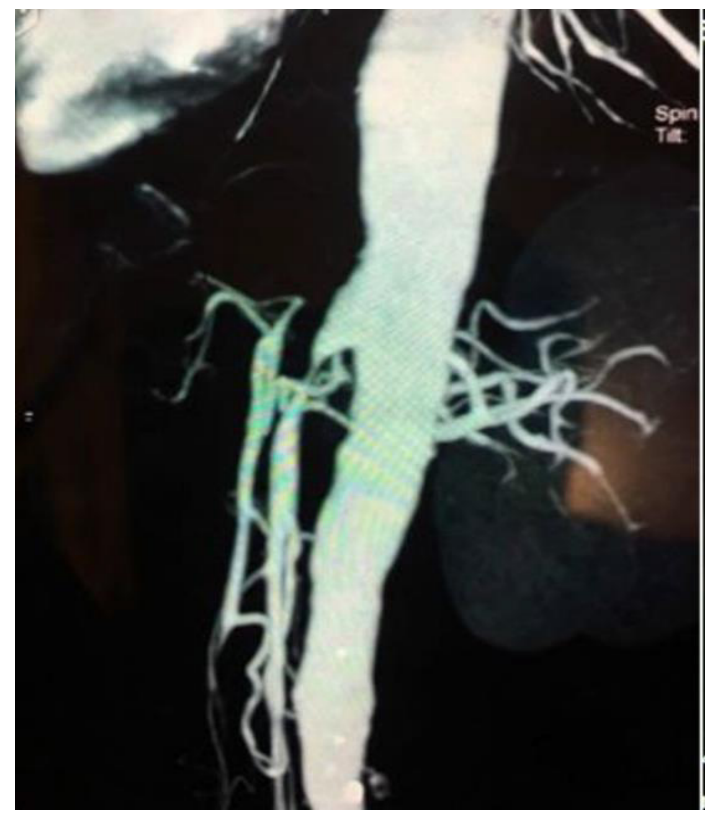

A

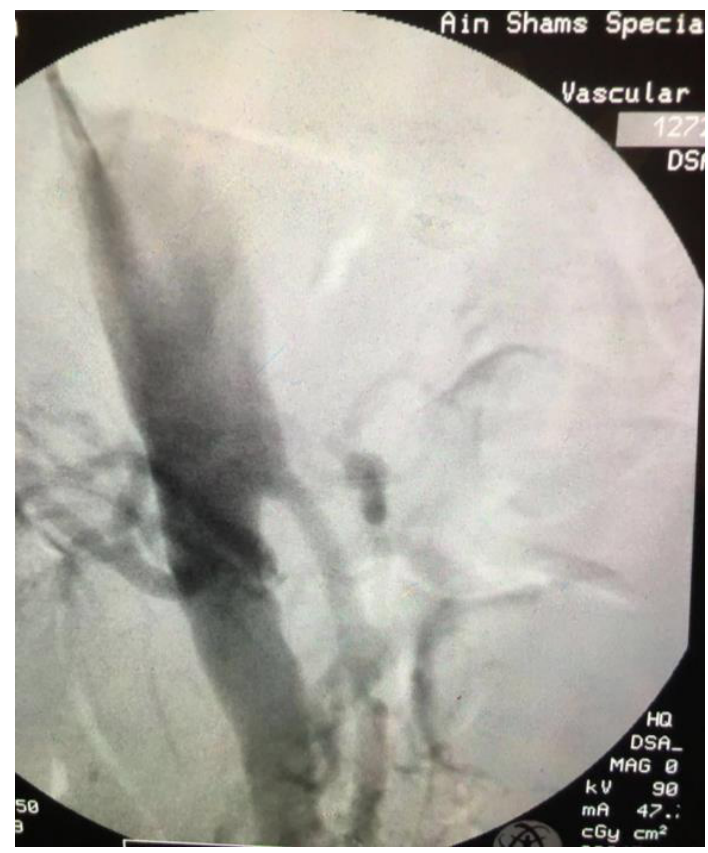

B

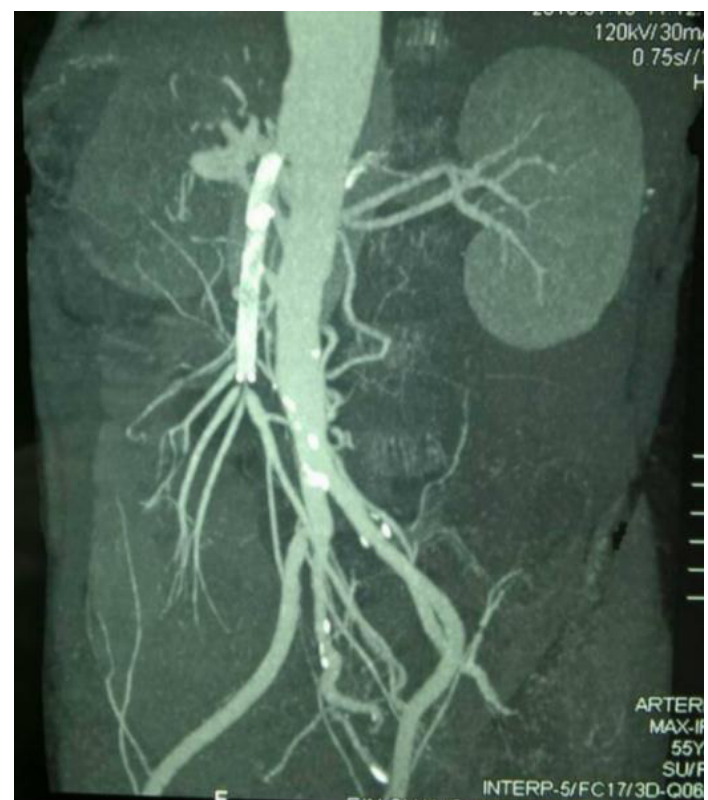

C

Fig 1. CT angiography of a patient that presented with postcibal angina of 2 years duration that was complicated with weight loss. CTA showed occlusion of the Celiacand the Inferior mesenteric arteries with stenosis in proximal superior mesenteric artery (A). Balloon dilatation and stenting of SMA was done (BMS + SES) (B). The patient had marked postoperative improvement and regained his usual body weight. Eleven months later, symptoms returned, and CTA showed intimal hyperplasia at the distal end of stents (C). Dilatation with DEB was done (not shown), with postoperative improvement. Till the end of the follow up period, the patient was symptom free.

\section{Results}

Data was obtained for 7 patients with chronic occlusive mesenteric ischemia involving the coeliac and superior mesenteric vessels, they were offered selective angiogram with/ without single level correction. Demographic and clinical features of the study group are shown in Tables 1,2. The group consisted of $5(71.4 \%)$ males and $2(28.6 \%)$ female with a mean age of $58.8 \pm$ 7.4 years. 4 patients were having diabetes mellitus, all patients were under treatment for hypertension, 6 patients were smokers, whereas 4 patients had a history of cardiac disease and all patients were hyperlipidemic. 
Regarding the presentation, $6(85.7 \%)$ patients were presented by weight loss, 4 (57.1\%) patients had postcibal angina, $6(85.7 \%)$ suffered from vague abdominal pain, and $2(28.6 \%)$ had anemia and 2 $(28.6 \%)$ of them were associated with gastric ulcers, as shown in Table 2.

Trial of angioplasty was done for the 7 patients, targeting to revascularize single vessel mainly the superior mesenteric artery (SMA). However, we failed to cross the lesion in one patient, and he was offered a surgical option. Four patients had ostial SMA lesions and were treated with primary stenting by balloonmounted stents (BMS), one patient with lesion not involving the ostium were treated with primary stenting by self-expandable stents (SES). One patient had ostial and non-ostial SMA lesions; both types of stents were used. Two patients had lesions involving celiac artery and were treated by balloon dilatation without stenting for one of them and the other one with primary stenting by BMS. All Patients were followed up and clinical improvement was recorded.

Immediate technical success was achieved in 6 patients (\%), as shown in Table 3. Clinical success was achieved in 5 patients, while one patient still had mild pain and discomfort and improved within 1 month. All the patients had follow up for $1,3,6,12,18$, and 24 months clinically and by either mesenteric duplex or CT mesenteric angiogram when recurrence is suspected.

At 6 months, one patient needed further management because his condition got worse because of having tight in-stent stenosis. Dilatation with Drug coated balloon was effective to relieve the stenosis and improve the patient's symptoms. 12 months later, the symptoms recurred due to in-stent restenosis. Revascularization was done with Drug coated balloon (DCB), and the patient was symptom free during the follow up period (24 months since the index procedure).

Two (28.6\%) patients continued to improve till the $9^{\text {th }}$ $11^{\text {th }}$ month of their follow up, both needed reintervention after 12 months. One patient had in-stent stenosis that was treated with $D C B$, and the other had total occlusion of the stent due to intimal hyperplasia at the distal end of the stent; and was treated with DCB and SES distal to the previously stented lesion. One patient was symptom free for 14 months and received dilatation with $D C B$ at the 15th month. The last patient was symptom free for 20 months, and then, he required revascularization with $\mathrm{DCB}$.

No major complications occurred in our series. However, minor complications occurred to 2 patients. The first had moderate postoperative hematoma at the cubital fossa (after transbrachial access). This was managed conservatively with complete resolution within 2 weeks. The other patient had femoral arteriovenous fistula. He was managed conservatively, and closure of the fistula was documented radiologically after 3 weeks.

All patients remained symptom free till the end of the follow up period (24 months since the index procedure). All reinterventions were due to intimal hyperplasia within the stent or at its distal end.

Table 1: Demographic data

\begin{tabular}{ll}
\hline Characteristics & N (\%) \\
\hline Age & $49-72$ (mean 58.8 years) \\
Smoking & $6(85.7 \%)$ \\
Diabetes & $4(57.1 \%)$ \\
Ischemic Heart disease & $4(57.1 \%)$ \\
Hypertension & $7(100 \%)$ \\
Hyperlipidemia & $7(100 \%)$ \\
Previous abdominal operations (for non-vascular cause) & $2(28.6 \%)$ \\
\hline
\end{tabular}

Table 2: Clinical Data

\begin{tabular}{ll}
\hline Postcibal pain & $4(57.1 \%)$ \\
Vague abdominal pain & $6(85.7 \%)$ \\
Weight loss & $6(85.7 \%)$ \\
Associated gastric ulcer & $2(28.6 \%)$ \\
Anemia & $2(28.6 \%)$ \\
\hline
\end{tabular}




\section{Access site}

Transfemoral

Transbrachial

$6(85.7 \%)$

\section{Vessels affected}

Celiac

Superior mesenteric

Inferior mesenteric

SES for SMA

BMS for SMA

SES + BMS for SMA

Balloon only for SMA 0

SES for celiac artery 0

Balloon celiac

BMS for celiac artery

Technical success

BMS = Balloon-mounted stent, SES= Self-expandable stent, SMA= Superior mesenteric artery.

Table 4: Post-procedural follow up

\begin{tabular}{ll}
\hline Access site complication & $2(28.6 \%)$ \\
Hematoma & $1(14.3 \%)$ \\
Fistula & $1(14.3 \%)$ \\
Vessel primary assisted patency (months) & $12-20($ mean 16.2 months) \\
Recurrence & $5(71.4 \%)$ \\
Restenting & $1(14.3 \%)$ \\
\hline
\end{tabular}

\section{Discussion}

Open revascularization has been the best treatment for low-risk patients due to durability and efficacy. Endovascular revascularization for chronic mesenteric ischemia was primarily indicated for elderly and higherrisk patients, but this has changed due to development of more precise devices and lower morbidity and mortality rates despite the higher recurrence and restenosis rates. ${ }^{6}$

Increasing acceptability of catheter-based therapies in other vascular territories along with accumulating experience in the mesenteric vessels are rapidly making angioplasty and stenting the first line therapy of choice for CMI. ${ }^{7}$

In this study, we report the clinical presentation and the results of endovascular management in 7 patients presenting with chronic mesenteric ischemia in Ain Shams University hospitals during the period between March 2014 and October 2016.

Our experience with mesenteric ischemia confirms that endovascular therapy is a safe and effective treatment modality. Procedural success was $85.7 \%$ and rate of minor periprocedural complications was $28.6 \%$, no major complications occurred. In addition, long-term results are favorable since the 2 year primary assisted patency is $71.4 \%$.

The main limitation to this study is the small number of patients which restricts our ability to extract statistically significant results. This shows the importance of developing a screening program to detect patients with chronic mesenteric ischemia.

\section{Conclusion}

Endovascular surgery is gaining in popularity for the treatment of atheromatous chronic mesenteric ischemia because of its less invasive nature and the reduced perioperative morbidity and mortality in comparison with open surgery. Superior mesenteric artery should be the main target of revascularization due to its massive collateralization with branches of both celiac and inferior mesenteric arteries. Drug coated balloons are important adjuncts to mesenteric angioplasty, and 
they appear to prolong the assisted primary patency to the stented arteries. Strict postoperative follow up is mandatory.

\section{References}

1. Kolkman JJ, Geelkerken RH: Diagnosis and treatment of chronic mesenteric ischemia: An update. Best Pract Res Clin Gastroenterol. 2017; 31(1) :49-57.

2. Barret M, Martineau C, Rahmi G, Pellerin O, Sapoval M, Alsac JM, Fabiani JN, Malamut G, Samaha E, Cellier C: Chronic Mesenteric Ischemia: A Rare Cause of Chronic Abdominal Pain. Am J Med. 2015; 128(12): 1363 .

3. Hawkins BM, Khan Z, Abu-Fadel MS, Exaire JE, Saucedo JF, Hennebry TA: Endovascular treatment of mesenteric ischemia. Catheter Cardiovasc Interv. 2011; 78(6): 948-52
4. Sarda P, Kumar G, Gupta D, Sanghvi S, Baroopal A: Endovascular management of chronic mesenteric ischemia: A single centre experience in Western Rajasthan, India. Int J Res Med Sci. 2017; 5(6): 2572-2574.

5. Gibbons CP, Roberts DE: Endovascular treatment of chronic arterial mesenteric ischemia: a changing perspective? Semin Vasc Surg. 2010; 23(1): 47-53.

6. Oderich GS, Malgor RD, Ricotta J] $2^{\text {nd }}$ : Open and endovascular revascularization for chronic mesenteric ischemia: tabular review of the literature. Ann Vasc Surg. 2009; 23(5): 700-12.

7. Razavi M, Chung $\mathrm{HH}$ : Endovascular management of chronic mesenteric ischemia. Techniques in Vascular and Interventional Radiology. 2004; 7(3): 155-159. 